TABLE V

\begin{tabular}{|c|c|c|c|c|}
\hline $\begin{array}{c}\text { Opinion } \\
\text { of the Evidence }\end{array}$ & Smokers & $\begin{array}{l}\text { Former } \\
\text { Smokers }\end{array}$ & $\begin{array}{c}\text { Non- } \\
\text { smokers }\end{array}$ & Total \\
\hline $\begin{array}{l}\text { Satisfied .. } \\
\text { Not satisfied } \\
\text { Undecided }\end{array}$ & $\begin{array}{r}46 \\
45 \\
9\end{array}$ & $\begin{array}{r}30 \\
12 \\
3\end{array}$ & $\begin{array}{r}34 \\
5 \\
9\end{array}$ & $\begin{array}{r}110(56 \cdot 5 \% \\
62(32.5 \%) \\
21(11.0 \%)\end{array}$ \\
\hline
\end{tabular}

unsatisfied with the evidence, a ratio of 1.3 to 1 . In group (2) the figures are 34 and 5, a ratio of 6.8 to 1 in favour of accepting the evidence. Thus, excluding those who were undecided, over five times as many non-smokers as present and former smokers accepted the evidence.

Of the 46 smokers who do accept the evidence 14 hope to discontinue smoking and 32 do not intend doing so.

\section{Discussion and Conclusions}

Less than half of this small group of doctors smoke cigarettes, a lower proportion than among the general population. The proportion of those who have never smoked $(27.6 \%)$ corresponds closely to Doll and Bradford Hill's (1954) finding of $28.5 \%$ of non-cigarette smokers among male doctors aged 35-44.

In the present group of 211 doctors the number who smoke cigarettes is gradually declining. Eight years ago it was 129 , now it is 100 .

To judge from the reasons which those who have discontinued smoking give for doing so and from figures of national tobacco consumption it appears that increased cost is a considerable factor in reducing cigarette smoking, but that the risk of cancer is not (although it may be a deterrent to starting the habit). This is supported by the fact that the number of doctors giving up smoking since an association with bronchial carcinoma was discovered has not increased. It seems, therefore, that further demonstration of this association will not lead to many more doctors stopping smoking.

The proportion of those who accept the evidence of an association between smoking and carcinoma of the bronchus is so much higher among the non-smokers than among the smokers, present and past, that personal prejudice may have played some part in shaping these opinions. Possibly the pleasures of tobacco addiction persuaded some of the smokers to discount the evidence of its dangers.

\section{Summary}

The 217 doctors working in the hospitals of Oxford were questioned about their cigarette-smoking habits, and $211(97 \%)$ replied : $47.4 \%$ smoke cigarettes, $25.1 \%$ once smoked, and $27.5 \%$ have never done so. The proportion who smoke is gradually declining, but the number of doctors giving up smoking has not increased since the discovery of an association between smoking and carcinoma of the bronchus. Figures for national tobacco consumption and number of smokers have shown no decline since 1950, when Doll and Bradford Hill's first paper appeared.

Only a little over half $(56.5 \%)$ of the doctors were satisfied with the evidence linking carcinoma of the bronchus and smoking; but the proportion was more than five times higher among the non-smokers than among the smokers. Perhaps the smokers were persuaded by their fondness for tobacco to discount unpalatable evidence.

I am most grateful to the 211 doctors for their co-operation and to Dr. A. M. Cooke for his criticisms.

\section{REFERENCES}

Doll, R., and Hill, A. Bradford (1950). British Medical Journal, 2, 739. - — (1954). Ibid., 1, 1451.

\section{PHYSIOLOGICAL PRINCIPLES IN THE TREATMENT OF THE UNCONSCIOUS PATIENT*}

BY

\author{
WILLIAM W. MUSHIN, M.A., M.B. \\ F.F.A. R.C.S., D.A.
}

Professor of Anaesthetics, Welsh National School of Medicine, Cardiff

The title of this paper crystallizes the main concern of the anaesthetist so far as the administration of general anaesthesia is concerned. He deliberately administers combinations of powerful drugs to produce unconsciousness of varying depth. His skill lies not only in the administration of these drugs, but in the treatment of the resulting unconscious state. The essential problems attendant on unconsciousness per se are the same whatever the cause, be it anaesthesia or head injury or barbiturate overdose. There therefore seems to be some justification for extending the discussion beyond the confines of anaesthesia to include unconsciousness in generat. As for the physiological principles which underlie the treatment of the unconscious state, the need for them here is no less than in the treatment of other pathological states-indeed more, since a disregard of such principles so easily leads to permanent damage or death.

\section{What is Unconsciousness?}

"Unconsciousness" is a term applied to three separate states which have much in common with each other and yet differ fundamentally in their aetiology and prognosis. They are natural sleep, anaesthesia, and coma. In each of these the patient loses his power of perception of the world in which he lives, and on recovery has no memory of events which took place during that time. Though he may respond in a reflex manner to stimuli, such reflex activity leaves no trace in his conscious memory. Natural sleep is distinguished by the fact that it occurs in the absence of disease or the administration of drugs. It terminates spontaneously after a varying interval, but in any case is easily interrupted by the application of external stimuli of very moderate intensity. Anaesthesia differs from coma only in the fact that it is to a great extent reversible and controllable by the anaesthetist. It is the result of the deliberate administration of drugs or of physical agencies such as cold or electrical currents. The term " coma" is best reserved for states of unconsciousness assọciated with disease, injury, or overdose with certain drugs. It may on occasion be reversible, but it is generally not controllable. The two differ little so far as the physical state of the patient is concerned, and it would be generally impossible on clinical examination alone, and withouf knowing the circumstances, to say whether the state is one of anaesthesia or of coma.

The depth of unconsciousness may vary widely from. patient to patient, and is determined by the individual ability to respond to stimuli of varying intensity. When a patient responds reflexly to mild painful stimuli his. unconsciousness is said to be light, but in profound unconsciousness it may be that no stimulus of any kind produces a response and only a beating heart and active respiration show that the patient is still alive.

*Based on a lecture given before the Royal Faculty of Physicians and Surgeons of Glasgow on January 19, 1955. 


\section{Why Treatment is Needed}

It might appear that the unconscious patient needs no treatment apart from the removal of the causes of his unconsciousness, whereupon he would return to consciousness and recover. This is certainly true in a number of instances, but there is a great risk that in the absence of positive treatment certain complications may arise which may be fatal or leave permanent effects.

\section{Respiratory Depression}

The very state of unconsciousness is outward evidence of a depressed activity of the cerebral cortex. Concomitant with this there is always depression of the rest of the brain, including the medullary centres. Respiration is therefore also depressed. Even more important than the reduction in the total minute volume of respiration is the reduction in tidal volume. When the size of the latter approaches that of the physiological dead space, hypoxia is present in some degree and the patient becomes cyanosed. This is because, in these circumstances, at each breath exchange of air takes place only between the dead space and the outer air. A good example of this is the patient who has tachypnoea due to an overdose of trichlorethylene. The respiratory rate may be $40-50$ per minute, but, since at each breath no more than perhaps $200 \mathrm{ml}$. of air is inhaled, cyanosis is present, even though the minute volume of respiration is high-10 litres or more per minute. Another example is the depression that occurs after overdosage with morphine or a barbiturate drug. Not only is the respiratory rate slowed, but the breathing is very shallow. When the volume of each inspiration is reduced to $150-200 \mathrm{ml}$. the patient usually becomes cyanosed. This explains why there is a limit to the slowing of respiration before death supervenes. Even were the patient taking good-sized breaths of $500 \mathrm{ml}$., at least three or four of them every minute would be required to give him enough oxygen. It is therefore impossible for such a patient to breathe at less than, say, four times a minute and live. Periodic breathing is common in unconscious patients, and is further evidence, if such were needed, of suboxygenation of the medulla:

\section{Respiratory Obstruction}

The respiratory depression of the unconscious patient may be, and often is, complicated by obstruction of the airway, either by a relaxed tongue and pharynx or by secretions or vomit. Secretion in the respiratory tract appears very quickly in the presence of respiratory obstruction, and such fluid is not salivary in origin. In these circumstances the secretion is generally the result of some degree of what is known, for want of a better term, as pulmonary oedema. The high intrapulmonary negative pressure consequent on respiratory obstruction upsets the delicate equilibrium at the alveolar membrane which keeps fluid from entering the alveoli from the blood stream. Liquid then easily passes from the blood stream into the alveoli and thence into the respiratory tract. (See under “Complications.")

\section{Circulatory Depression}

Treatment is also needed because of the disturbances of circulation. Particularly in anaesthesia, but also in many other states, the heart muscle itself is depressed, and in any case will soon become so when hypoxia develops. The action of the heart is further hindered by the depressed breathing. The "thoracic pump" effect of breathing on the venous return of blood is lessened, the output of the heart is reduced, and the blood pressure tends to fall. At the other end of the circulatory system the capillaries are affected by hypoxia and by the accumulation of metabolic products, which quickly follow in the train of depressed breathing. Peripheral resistance is lowered and the blood pressure tends to fall still further. A vicious circle is started : poor cardiac output and low blood pressure mean poor pulmonary circulation and poorer oxygenation; the lower blood pressure ultimately means less coronary flow and still further depression of the heart muscle.

\section{Other Effects}

Metabolism is generally lowered during unconsciousness. Water loss, too, occurs continuously, and ultimately with the passage of time the problem of water balance and nutrition arises. There are certain other problems, minor if guarded against, but major in their possible ill-effects. Two examples are the possibility of the inhalation of vomit and the possible development of nerve palsies through malposture.

If untreated, therefore, the unconscious patient develops increasing hypoxia, with tissue and pulmonary oedema and circulatory failure. His metabolism is lowered and his fluid balance disturbed; secretions and vomit may be inhaled and postural palsies develop.

\section{Treatment of the Unconscious State}

The first principle of treatment of the unconscious patient, and one on which all else depends, is the maintenance of an adequate supply of oxygen to the tissues. Air or oxygen must first reach the alveoli in sufficient amount, and then enter the blood stream. I am sometimes shocked that this elementary fact is not appreciated outside the field of anaesthesia. My last experience of this was in connexion with a case labelled "cerebral malaria," to which I had been called because an oxygen regulator was out of action. The toothless patient, with a hanging lower jaw, was propped up in bed, unconscious, cyanosed, and clearly dying. Each breath was made with effort, and the sucking-in of his chest was striking. I immediately held up the patient's jaw, and to the amazement of those present the stertor disappeared, the patient became pink, and within a few hours was fully conscious. He ultimately left hospital a fit man.

If the jaw cannot be held in such a way that breathing is free for long periods of time, or if the unconsciousness is profound, an endotracheal tube should be inserted without hesitation. The insertion of a tube into the trachea should no longer be regarded as requiring unusual skill or as carrying great hazard. The manœuvre is performed daily by anaesthetists, and is a life-saving measure when the patency of the airway cannot be maintained by other means. The introduction of the practice of intubating seriously ill patients with respiratory insufficiency in spinobulbar poliomyelitis, and more recently in tetanus, has had a dramatic effect on the prognosis of these diseases. I believe that a more widespread appreciation of the importance of a perfect airway will lead to a greater use of tracheal intubation with good results. If the unconsciousness is likely to persist for.more than one or two days a tracheostomy is preferable to an endotracheal tube, since trauma to the cords is avoided and the respiratory tract is more easily kept free of secretions.

\section{Suction}

An important accessory aid to a clear airway is the use of mechanical suction. There can be few hospitals without a suction apparatus, and one of these instruments must also be regarded as essential for domiciliary use. Without going too deeply into mechanical matters, I would like to point out that the "power" or "strength" of the apparatus refers to the rate at which it removes fluid. The negative pressure indicated on a manometer is by itself of little interest. A good hypodermic syringe can produce almost a vacuum, but is useless as a "sucker" because its liquid removal rate is maintained for only a brief moment. The accompanying chart gives the results of some experiments with a piped suction installation, and illustrates some further points. For the removal of vomit or secretion a mechanical sucker is unequalled. Even with an efficient sucker, and certainly in its absence, gravity can still save life. A headdown position allows vomit to fall to the floor and prevents its accumulation in the pharynx, whence it may be inhaled.

\section{Carbon Dioxide}

Even when the airway is perfectly clear hypoxia may still be present owing to shallow breathing. The administration 
of oxygen helps in these cases, but until ventilation has increased and tidal exchange become larger inadequacy of oxygenation may persist. Shallow breathing is a temptation to use carbon dioxide. But poor ventilation itself results in an accumulation of carbon dioxide, and the depressant effects of this gas on the central nervous system and the myocardium are now being appreciated to a much greater degree than formerly. The carbon-dioxide content of the blood of a depressed unconscious patient is generally high,

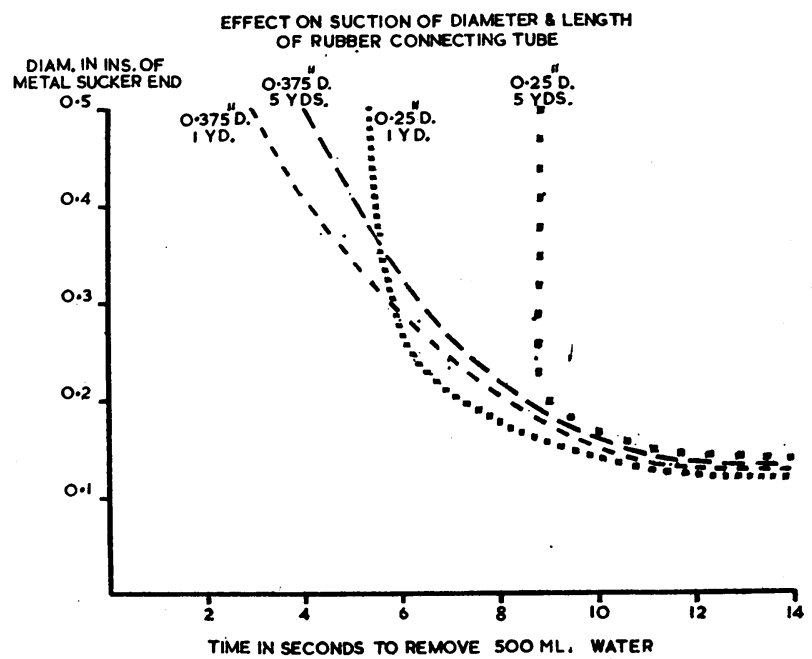

Chart showing the results of experiments with a piped suction installation. In the case of small-bore tubing, increasing the diameter of the metal sucker end has little effect in improving the fluid-removal rate. Improvement is effected by shortening the connecting tubing. The best results are obtained with large-bore connecting tubing and large-bore metal sucker end.

and more than enough to stimulate the respiratory centre if only the sensitivity of the centre is restored by an adequate supply of oxygen. Carbon dioxide from a cylinder plays little or no part in the treatment of respiratory depression. There is, indeed, a grave danger attached to its use, since a concentration above $7 \%$ has a powerful depressant effect on the central nervous system; in fact a high concentration was at one time deliberately used as an anaesthetic.

\section{Oxygen}

The average patient produces between 200 and $300 \mathrm{ml}$. of carbon dioxide per minute, and this must be expelled through the respiratory tract. At least 5 to 6 litres of other gases is required if this amount of carbon dioxide is to be diluted to $5 \%$, the normal concentration in the alveoli. If oxygen therapy is instituted, at least 6 litres of oxygen must flow into the bag or tent. A smaller amount than this cannot possibly produce a sufficient partial-pressure differential of carbon dioxide across the alveolar membrane for the expulsion of the full amount of the gas.

\section{Analeptics}

An old-fashioned way of treating narcotic poisoning was to slap the patient's face and drag him round the room; another was to dilate the anal sphincter. The aim of these and similar methods, including the injection of analeptics, is to stimulate breathing reflexly. I cannot, however, support or encourage such methods. When a patient is deeply depressed, only oxygenation of his vital organs, particularly the brain, could keep him alive. Analeptics and physical stimuli are generally useless in such cases. Their continued administration when they fail to produce response is usually evidence of lack of appreciation of the depth of depression of the patient's central nervous system. It should be borne in mind, however, that spontaneous respiration. in the presence of anoxia may be largely the result of stimulation of the carotid body by anoxia. Oxygen overcomes this, but if the respiratory centre is too depressed for carbon-dioxide stimulation to be continued apnoea may result. Such a patient may need assisted respiration until his respiratory centre recovers sufficiently from its depression.

\section{Artificial Respiration}

An excellent method of maintaining artificial ventilation of a depressed patient for long periods of time is the box or cabinet respirator. The fact that a sub-atmospheric pressure is produced round the body with this type of apparatus should not obscure the similarity between it and rhythmic positive-pressure ventilation. In both the essential feature is the creation of a differential between the pressure at the lips and that in the chest. It makes little gross difference to the respiratory or circulatory mechanics whether that differential is produced by raising the air pressure round the head or by lowering it round the body.

Of the numerous methods of artificial respiration that are still in common use, the only one suitable for emergency clinical use is inflation of the lungs. There was at one time among physicians a general suspicion of inflation of the lungs with air or oxygen at a pressure above that of the atmosphere, on the ground that such a pressure may injure the alveoli. It should be remembered in this connexion that when the procedure is carried out with a thin rubber bag the maximum pressure attainable is of the order of $30-40 \mathrm{~cm}$. of water. This is far below the intrapulmonary pressure of over $100 \mathrm{~cm}$. of water which can be voluntarily generated by closing the laryn $x$ and forcefully expiring. It is likely that a pressure much in excess of $150 \mathrm{~cm}$. of water is required before alveolar damage occurs. None of the foregoing would, of course, exclude the risk of rupturing an unsuspected subpleural bulla, but this risk is present in everyday life quite apart from artificial respiration. There are, however, certain disadvantages of positive-pressure inflation of the lungs which should be known, though they are generally insignificant as compared to the advantages. There is, first, a possibility of occluding the alveolar vessels at the peak of each inflation. To avoid this the mean inflation pressure should be as low as possible, with peaks not in excess of $15-20 \mathrm{~cm}$. of water, and even this pressure should not be maintained for more than a fraction of the cycle. A sharp peak to the pressure curve rather than a plateau is the objective. The other risk is that too vigorous, and particularly too frequent, a rhythm of positive pressure may so tamponade the heart and impede the normal return of venous blood by the thoracic pump that the cardiac output is impaired. Prophylaxis here consists in applying a slow rhythm and allowing a rapid return of the intrapulmonary pressure to that of the atmosphere at the end of each inflation. These measures ensure that the mean intrapulmonary pressure is low and the circulatory impedance as low as possible.

\section{Posture}

The common practice of placing an unconscious patient flat on his back exposes him to the hazards of respiratory obstruction and of inhaling vomit and secretions. A better posture, and one which to a large extent overcomes these risks, is to place him on his side. Vomit and secretions collect in the cheek and, if there is a slight head-down tilt, will run out, while the flaccid tongue falls to one side and the airway does not become obstructed. At regular intervals, say once an hour, the patient should be turned to the other side to encourage good circulation on the two sides of the body to improve venous return.

\section{Maintenance of Fluid Balance}

In an unconscious patient it is essential that the fluid and electrolyte balance be maintained. Only losses should be replaced. The accurate and careful charting of fluid balance should be started at the earliest moment, regard being paid to the importance of vomit and sweating as sources of fluid loss. Nutrition becomes a problem only in unconsciousness lasting more than a couple of days; owing to the low metabolism in such cases a daily intake of about 1,500 calories is generally adequate. 


\section{Complications}

\section{Resistance to Breathing}

In discussing the treatment of unconsciousness attention should be drawn to the importance of minimizing the resistance to breathing imposed by the use of such devices as endotracheal tubes and oxygen-therapy apparatus. It is essential that a patient, particularly one whose respiration is depressed, should not be required to make more effort to breathe than is necessary. If such effort is called for the patient becomes physically exhausted, and if the effort required is too great he may be incapable of making it. As a result the lungs are not filled quickly enough and oxygen intake is lowered. In addition, the intrapulmonary negative pressure at the height of inspiration may be greatly increased and the path prepared for pulmonary oedema. With an endotracheal tube, or any other gas pathway through which the patient is made to breathe, the factors which influence resistance are the diameter and length of the instrument and the presence of sharp corners-all being equally important. In any case, the use of endotracheal tubes, invaluable though it is in preventing respiratory obstruction, does not mean that further obstruction cannot occur. Kinking at the back of the pharynx and the passage of a tube into one bronchus are not infrequent and must be watched for.

\section{Pulmonary Oedema}

A common complication of the altered mechanics of respiration in the unconscious patient is pulmonary oedema. Most physicians and anaesthetists have had experience of the speed with which moist sounds, and even frothy sputum, appear in the respiratory tract of such a patient. To understand why pulmonary oedema readily appears in these circumstances it is necessary to indicate briefly the normal equilibrium across the alveolar membrane which keeps liquid within the circulatory system. This consists of a balance of forces. On the one hand there is the pressure within the alveoli, falling a little at each inspiration and returning to atmospheric pressure during expiration; on the other side of the membrane is the vascular system. A filtration force of $10 \mathrm{~mm}$. $\mathrm{Hg}$, the systolic pressure, tries to force fluid out into the alveoli, while the osmotic pressure of the blood proteins, of about $20-25 \mathrm{~mm}$. $\mathrm{Hg}$, acts in the reverse direction. When respiratory obstruction is present the intra-alveolar pressure falls unduly at each inspiration, tending to expel fluid. This is further enhanced if the patient has a right heart failure of any degree, leading to a high pulmonary arterial pressure, or has been injudiciously given blood transfusion. or saline infusion. If in addition there is a general anoxia, the alveolar cells and the alveolar vessels also become anoxic, their permeability is increased, and a vicious circle created. Suction can clear fluid only from the trachea and main bronchi; it cannot empty the alveoli. Positive-pressure ventilation with an unobstructed airway can reverse this process. Confirmation of this view may be found in the growing practice nowadays of treating the respiratory depression of barbiturate overdose, the respiratory paralysis of poliomyelitis, and also the paralysis induced by curare in cases of tetanus, by means of tracheostomy, positive-pressure ventilation, and frequent suction. In thęse instances oxygenation is ensured, the respiratory tract kept clear, and pulmonary oedema prevented.

\section{Summary}

An outline is given of the main physiological problems common to unconscious patients whatever may be the underlying cause, and in particular those of interest to an anaesthetist.

Treatment resolves itself into ensuring adequate oxygenation by means of a clear airway, with some form of assisted or artificial respiration where indicated. The improved circulation, with fluid loss made good, combines with the improved oxygenation and respiratory mechanics to prevent the altered dynamics across the alveolar membrane which leads to pulmonary oedema and may prove fatal. Many an unconscious patient who has been adequately treated for the underlying cause dies unnecessarily because these fundamental physiological requirements are not met.

\section{TREATMENT OF LEUKAEMIA AND ALLIED DISORDERS WITH 6-MERCAPTOPURINE}

BY

JAMES R. FOUNTAIN, M.B., M.R.C.P.Ed.

Medical Tutor, Department of Medicine, the General Infirmary at Leeds

Within the past ten years a number of chemical agents which produce temporary beneficial effects in leukaemia and allied diseases have been discovered. Their introduction has usually come about in one of three ways : (a) the observation that a compound being investigated for other reasons has a depressant effect on haemopoietic tissues-for example, nitrogen mustard and urethane; $(b)$ the synthesis of compounds related chemically to substances already known to be effective, such as in the case of triethylene melamine, triethylene phosphoramide, and naphthyl-di-2-chlorethylamine (R.48); and (c) the synthesis of analogues of nucleic-acid precursors. It is on the last group that so much attention is now being focused. Undoubtedly the observation by Farber et al. (1948) that two folic-acid conjugates, pteroyl-diglutamic acid and pteroyl-triglutamic acid when given to children with acute leukaemia resulted in an acceleration of the leukaemic process gave considerable impetus to research in this particular field. The sequel was the synthesis of the 4-amino analogues of folic acid, aminopterin and amethopterin, which were found to suppress the growth of leukaemic tissue and produce temporary remissions in some children with acute leukaemia (Proceedings of the Second Conference on Folic Acid Antagonists, 1952).

With the knowledge that folic acid was essential for the synthesis of nucleic acid, antagonists of other nucleic-acid precursors have been prepared and tested for antileukaemic activity. 2:6-Diaminopurine, an analogue of adenine, has been shown (Burchenal, Bendich, et al., 1949 ; Burchenal, Johnston, et al., 1949) to prolong the survival time of mice with transplanted leukaemia. Burchenal, Karnofsky, et al. (1951) reported remissions in five of a series of 25 patients with acute leukaemia treated with this compound. Compounds containing the $2: 4$-diamino-pyrimidine moiety, including those with antimalarial activity (Falco et al., 1949), were shown to be competitive antagonists of folic acid in the growth of Lactobacillus casei (Hitchings et al., 1952), and in animals states indicative of folicacid deficiency were observed. These findings suggested that such compounds might be effective in acute leukaemia, and it has been shown (Burchenal, 1954) that one such pyrimidine analogue, $2: 4$-diamino-5 $\left(3^{\prime} 4^{\prime}\right.$-dichlorphenyl)-6-methylpyrimidine, produced occasional remissions in children with this disease. Farber, Foley, et al. (1953) have shown that a related series of compounds, the dihydrotriazines, have the property of temporarily inhibiting the growth of the leukaemic process in children. 